\title{
Otitis Media and the Impact on Children
}

\section{Lilian $\mathrm{F}^{*}$ \\ Assistant Professor, Lamar University, USA}

*Corresponding author: Lilian Felipe, Assistant Professor, Lamar University, USA, Email: lfelipe@lamar.edu

\section{Review Article \\ Volume 2 Issue 6}

Received Date: September 29, 2018

Published Date: November 01, 2018

DOI: $10.23880 / \mathrm{mjccs}-16000182$

\section{Abstract}

Background: Otitis media is one of the most common illnesses that affect children each year. Children under the age of six years old will likely have a middle ear infection at least once mainly due to anatomical structures and environment exposures to their immune system. While many cases of otitis media resolve spontaneously, there are many children who require medical treatment or have chronic difficulty with these infections. The increased recurrence of middle ear infections in a child can create a negative impact on their hearing, speech, and overall, learning abilities and development. Discussion: The impact of recurrent otitis media can include a persistent conductive hearing loss, speech delay, and delay in language development. These consequences can create a more challenging environment for children in school and may require additional services to provide help towards success in school. Additionally, recurrent otitis media may also affect children behaviorally and mentally. It is important to understand that the presence of these effects from otitis media is different for each child and circumstance. It is dependent on recurrence, severity, treatment, and seeking longterm help for learning.

Conclusion: Otitis media can affect anyone, but one of the most frequent populations is young children under the age of six years old. It is important to seek medical assessment and treatment as needed. If medical management is unhelpful, then seeking out help for management of long-term effects is key for child success in school.

Key words: Otitis Media; Children; Learning; Hearing Loss; Speech Delay

\section{Introduction}

Otitis media is an infection that occurs in the middle ear and can affect surrounding structures of the ear and auditory system. Otitis media is often referred to as a middle ear infection and is one of the most common illnesses that children experience [1-4]. During a middle ear infection, the tissue lining of the middle ear cavity becomes inflamed and fluid can begin to collect behind the tympanic membrane, or eardrum. While many bacteria, illnesses, infections, and genetics (i.e.
Strepococcus pneumoniae, influenza, upper respiratory infection, cleft palette, etc.) could trigger otitis media, the main cause of a middle ear infection is connected with the eustachian tube $[2,3]$.

The eustachian tube connects the throat, particularly the nasopharynx, to the middle ear cavity and functions by equalizing pressure in the middle ear cavity, protecting this cavity from external bacteria and debris, and clearing secretions from the middle ear cavity. The eustachian tube plays a vital role in functionality of the middle ear; 


\section{Medical Journal of Clinical Trials \& Case Studies}

therefore, when any illness or infection is present, bacteria can cause inflammation of the eustachian tube and cause the tube to malfunction. This eustachian tube dysfunction can create a buildup of negative pressure behind the eardrum and keep fluid within the middle ear cavity, instead of properly moving out air and secretions. Interestingly, eustachian tube dysfunction is more likely to occur in children due to the anatomical structure of their tube, shorter and more horizontal compared to the longer and more vertical adult's, and that children have a weaker, or uncompromised, immune system [3]. Most cases of otitis media fall under the category of acute otitis media [1-5].

This means that the infection may be a one- or twotime occurrence and severity may be mild or medically manageable. Many cases of acute otitis media, even in children, can resolve on their own with observation. However, more severe infections (such as presence of fluid, mucous, perforations of eardrum, or pain to surrounding areas) or frequently recurrent cases (over six months to a year) are likely reasons for medical management and/or classification of chronic otitis media. When middle ear infections are recurrent, medical management and seeking long-term help for management is crucial to minimize and negative effects that may come with repeated otitis media. This article aims to discuss the long-term effects related to recurrent otitis media and how treatment and management are essential for working towards success in school.

\section{Discussion}

Otitis media occurs commonly in early children. Research has shown that $80 \%$ of children experience otitis media by the age of 4 years [6]. Early onset has been associated with an increased probability of repeated episodes, which leads to long term effects. One long term effect of otitis media is a fluctuating mild-to-moderate conductive hearing loss. It can be present for a few weeks or persists for some months. This in turn affects speech and language development which can hinder educational abilities specifically in school age children.

When infections of the middle ear continue to occur, permanent damage can begin to take place in the auditory system. Scarring can occur along the tympanic membrane and the external auditory canal [3]. Deterioration of the ossicles and/or mastoid can occur in more severe cases of chronic otitis media. However, one the important areas of damage can occur to hearing. Acquired hearing loss can appear during otitis media, which can potentially cause some form of impact on speech and language development in children [1,2,4,5,7]. However, cases of recurrent or chronic otitis media can cause a permanent hearing loss in children, which truly impacts the ability to easily and properly develop speech and language. Researches insure to identify that the speech and language development difficulties occur due to the presence of a conductive, acquired hearing loss in most cases of acute otitis media and the chance of development into sensorineural, permanent hearing loss in severe and chronic otitis media cases $[1,2,4,5,7]$.

The first six years of life in a child sees the development of speech sounds. Taking any amount of time away from functional hearing for a child can damper the development of speech $[2,5,7]$.

\section{Role of Hearing in Language Development}

A mild to moderate fluctuating conductive hearing loss generally occurs due to continuous otitis media. This has been hypothesized to interfere with rapid language processing, which causes a child to encode information inefficiently, incompletely, or inaccurately [8].

Language is solely dependent of cognition and academic achievement, which is acquired almost exclusively through hearing [9]. If otitis media and the associated hearing loss persist or recurs during the formative years of language and learning, a delay in language development can occur, possibly affecting vocabulary or grammar [8]. In treating young children with otitis media, communicative skills are often ignored. Therefore, careful assessment should be made of language development or auditory-based learning disabilities in order to begin early intervention in children who are at risk. Research has shown that otitis media is a significant risk to speech production. If the auditory system is not exposed to enough sound due to a fluid-filled middle ear, it is not able to develop auditory synapses that allow us to process speech and language at a fast rate. As a result, children can experience difficulties forming strategies and rules in order to categorize and learn the acoustics of language. Aithal et al. supports this conclusion, stating that children with a history of otitis media have decreased linguistic experiences and decreased abilities to categorize speech stimuli as a direct result of their temporary increased thresholds [7].

Due to the increased amount of environmental noise in the classroom, school-aged children with otitis media will experience even more of a hearing handicap. Average 


\section{Medical Journal of Clinical Trials \& Case Studies}

classroom noise can mask voiceless consonants such as "p, t, k, s, sh, th, and f", making it nearly impossible for children with a mild conductive loss to hear and develop these consonants.

Studies have concluded that language comprehension, language expression abilities, speech articulation and reading scores were significantly lower in 5 years old children who experienced bilateral otitis media with effusion over those in the same age group that did not have a previous diagnosis [1,2,4,5,7]. Hutton (1984) reported that there is a higher incidence of children currently with or with a previous history of middle ear pathologies classified as learning disabled due to their lack of auditory stimulation as a young child. If otitis media is detected, diagnosed, and treated properly, children can avoid the adverse effects of the illness.

The most common treatment for otitis media is antibiotics. However, continued use of antibiotics can lead to resistance, resulting in little to no positive effects on treatment. The next step to treating chronic otitis media is the surgical placement of pressure equalization tubes within the tympanic membrane. Pressure equalization tubes help to maintain atmospheric pressure within the middle ear space, as well as properly drain fluid that may accumulate during or after an illness.

\section{Educational Impact}

Otitis media can negatively affect educational outcomes in children. If otitis media during preschool years affects auditory processing skills, children may find it difficult to process auditory information. If speech perception is affected, the awareness of sounds in words is also likely to be affected, leading to difficulties with reading and spelling. The preschool years are marked by an extremely rapid growth to the form and use of language [9]. This is the reason that the years between 1 and 5 are considered the critical or sensitive period for the acquisition of language and, as a consequence, the earlier in life that a recurrent hearing loss disrupts speech, the greater the risk of linguistic and subsequent cognitive disfunction.

The main consensus for medical management is use of antibiotics and surgical placement of pressure equalization tubes. Classroom amplification has been shown to reduce the impact of hearing, listening and attention deficits in the classroom. However, it is most effective when combined with analysis and modification of classroom acoustics. Children must also be taught effective listening behaviors.

It is important to highlight the multidisciplinary work of healthcare professionals, including schools, and how it is important that children have health administrators and school administrators on the same page of action.

\section{Conclusion}

Acute otitis media may resolve on its own, but it is important to understand that the recurrence of a middle ear infection increases the risk of developing negative effects to hearing, speech, language, and school. Treatment, specifically medical management, should always be sought out for infections of the ear, that way otitis media can be worked toward proper elimination with minimal negative functional impacts; however, it is important to know that functional impairments happen and can be addressed with helpful health and school providers and services that will work to bring children closer to success in school.

\section{References}

1. Fougner V, Kørvel-Hanquist A, Koch A, Dammeyer J, Niclasen J, et al. (2017) Early childhood otitis media and later school performance: A prospective cohort study of associations. Int J Pediatr Otorhinolaryngol 94: 87-94.

2. Medley LP, Roberts JE (1995) At-risk children and otitis media with effusion: Management issues for the early childhood. Topics in Early Childhood Special Education 15(1): 44.

3. Musiek FE, Baran JA, Shinn JB, Jones R (2011) Disorders of the auditory system. Plural Publishing.

4. Niclasen J, Obel C, Homøe P, Kørvel-Hanquist A, Dammeyer J (2016) Associations between otitis media and child behavioural and learning difficulties: Results from a Danish cohort. Int J Pediatr Otorhinolaryngol 84: 12-20.

5. Jensen RG, Koch A, Homøe P (2013) The risk of hearing loss in a population with a high prevalence of chronic suppurative otitis media. Int J Pediatr Otorhinolaryngol 77(9): 1530-1535. 


\section{Medical Journal of Clinical Trials \& Case Studies}

6. Williams CJ, Jacobs AM (2009) The impact of otitis media on cognitive and educational outcomes. Med J Aust 191(9): S69-72.

7. Aithal S, Yonovitz A, Aithal V (2008) Perceptual Consequences of Conductive Hearing Loss: Speech Perception in Indigenous Students Learning English as a 'School' Language. Australian \& New Zealand Journal of Audiology 30(1): 1-18.
8. Roberts JE, Rosenfeld RM, Zeisel SA (2004) Otitis media and speech and language: a meta-analysis of prospective studies. Pediatrics 113(3): e238-e248.

9. Eimas PD, Kavanagh JF (1986) Otitis media, hearing loss, and child development: a NICHD conference summary. Public Health Rep 101(3): 289-293. 